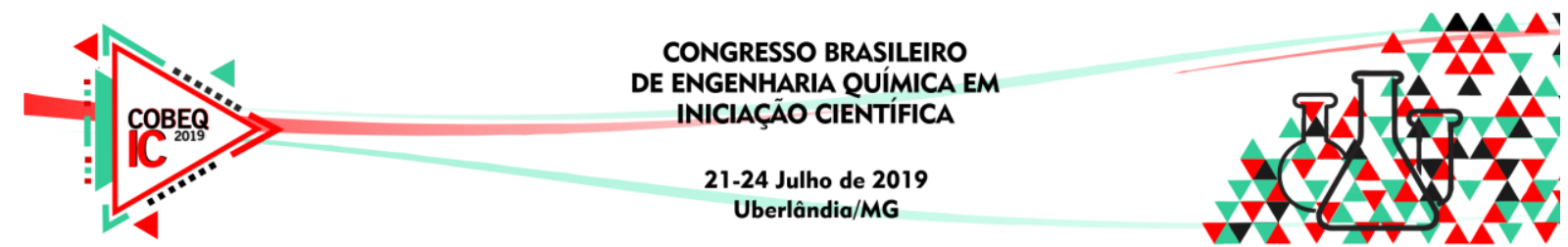

\title{
AVALIAÇÃO DA PRODUÇÃO DE ETANOL POR Saccharomyces cerevisiae SUPLEMENTADO COM FARELO DE ARROZ
}

\author{
M. A. GONÇALVES ${ }^{1}$, G. T. AMOGLIA ${ }^{1}$, D.E. B. DA SILVA ${ }^{1}$, M. G. SANTIAGO ${ }^{1}$, e F. \\ PALLADINO $^{1,2}$. \\ ${ }^{1}$ Pontifícia Universidade Católica de Minas Gerais, Departamento de Engenharia Química \\ ${ }^{2}$ Universidade Federal de Minas Gerais - Departamento de Biologia \\ E-mail para contato: ferpalladino12@gmail.com
}

\begin{abstract}
RESUMO - O emprego de subprodutos para a produção de etanol tem se mostrado cada vez mais presente no âmbito de pesquisas uma vez que busca-se reduzir os impactos gerados pelos combustíveis fósseis. Um exemplo disso é a utilização do farelo de arroz como suplemento no meio fermentativo para produção de etanol. Diante disso, o presente trabalho visa avaliar a produção de etanol por meio do uso de diferentes concentrações de farelo de arroz em conjunto com a variação de concentração de células e volume de meio utilizando a levedura Saccharomyces cerevisiae. As concentrações foram variadas em $\left(0-20\right.$ g. $\left.\mathrm{L}^{-1}\right)$ para o farelo de arroz, (1-10 g. $\left.\mathrm{L}^{-1}\right)$ para a concentração de células e $(25-75 \mathrm{~mL})$ para volume de meio, tais valores foram mensurados por meio de um planejamento fatorial $2^{3}$, com três pontos centrais. Foram calculados os parâmetros fator de conversão de substrato em produto, $\mathrm{Y}_{\mathrm{p} / \mathrm{s}}\left(\mathrm{g} \cdot \mathrm{g}^{-1}\right)$, produtividade volumétrica, $\mathrm{Qp}\left(\mathrm{g} . \mathrm{L}^{-1} \cdot \mathrm{h}^{-1}\right)$ e a eficiência do processo $\Pi(\%)$. Os resultados que apresentaram maior produção de etanol foram os ensaios 4 no tempo de 12 horas, com $10 \mathrm{~g} . \mathrm{L}^{-1}$ de células, $75 \mathrm{~mL}$ de meio e ausência de farelo de arroz, sendo a produção de etanol de 26,91 g. $\mathrm{L}^{-1}$ e o experimento 2 no tempo de 24 horas, com 1 g. $\mathrm{L}^{-1}$ de células, $75 \mathrm{~mL}$ de meio e ausência de farelo de arroz, sendo a produção de etanol de 26,36 g. $\mathrm{L}^{-1}$. De acordo com os resultados observou-se uma ótima produção de etanol nas condições avaliadas.
\end{abstract}

\section{INTRODUÇÃ̃}

Atualmente, além da preocupação em substituir o petróleo e da necessidade de se reduzir a emissão de gases poluentes, estudos têm sido realizados utilizando etanol de segunda geração como combustível mais sustentável (GONÇALVES, 2018).

O etanol pode ser produzido a partir da fermentação de vários tipos de matéria-prima, isto é, a partir da cana-de-açúcar, mandioca, milho, beterraba e entre outras. O processo de fermentação ocorre pela conversão de açúcares em etanol devido à ação de microrganismos. O microrganismo mais estudado para a produção de etanol é a levedura Saccharomyces cerevisiae, sendo esta a mais utilizada na produção em larga escala (GONÇALVES, 2018).

O controle do processo fermentativo é fundamental para atender a demanda energética do microrganismo envolvido no processo, sobretudo para uma conversão eficiente. Com isso, faz-se necessário conhecer as variáveis que influenciam o meio fermentativo, tais como $\mathrm{pH}$, temperatura, concentração de células, oxigenação, bem como nutrientes 


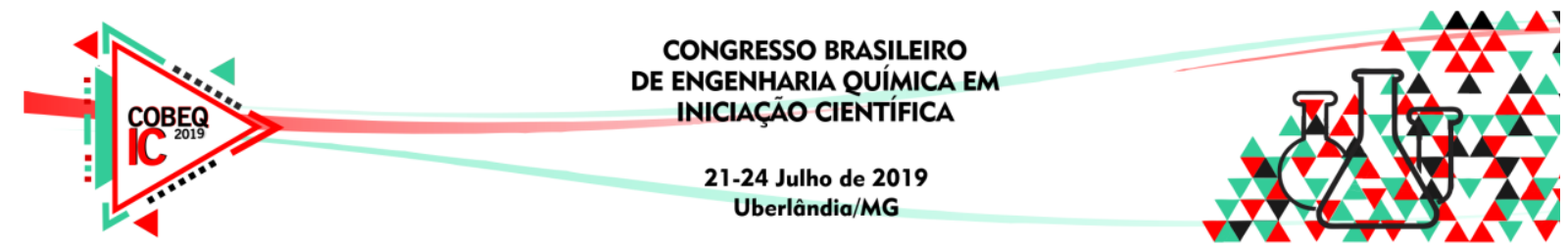

adequados que estimulem o desenvolvimento das células e a produção de etanol (FERRARI, 2013).

Com o avanço em pesquisas biotecnológicas, tem-se estudado como nutriente suplementar o farelo de arroz. O farelo de arroz é um subproduto da agroindústria rico em vitaminas do complexo B. Além das vitaminas do complexo B, o farelo de arroz possui em sua composição: vitamina $\mathrm{E}$, zinco, cobre, manganês, ferro e fósforo. A presença dessas vitaminas e desses minerais no farelo de arroz é essencial no meio fermentativo para o crescimento das células (WALTER; MARCHEZAN; AVILA, 2008).

Diante disso, o presente trabalho tem o intuito de avaliar a influência da concentração de farelo de arroz em conjunto com a concentração de células (Saccharomyces cerevisiae) e a concentração de oxigênio no meio fermentativo para a produção de etanol.

\section{METODOLOGIA}

Os experimentos foram realizados nos laboratórios de Biotecnologia e Genética e Análise Instrumental, pertencentes à Pontifícia Universidade Católica de Minas Gerais, campus Coração Eucarístico, na cidade de Belo Horizonte.

\subsection{Processo Fermentativo}

Para o processo fermentativo utilizou-se a levedura Saccharomyces cerevisiae, $e$ preparou-se as soluções estoque de glicose 200,0 g.L $\mathrm{L}^{-1}$, peptona 100,0 g.L $\mathrm{L}^{-1}$, extrato de levedura 100,0 g.L $\mathrm{L}^{-1}$ e sulfato de amônio 100,0 g.L $\mathrm{L}^{-1}$. Assim como, uma solução de farelo de arroz $200 \mathrm{~g} \cdot \mathrm{L}^{-1}$.

\subsection{Experimentos}

Os experimentos para o processo de fermentação foram realizados conforme o modelo de delineamento fatorial de $2^{3}$, utilizando três pontos centrais onde, as variáveis independentes são: farelo de arroz, concentração celular e concentração de oxigênio, sendo que a última foi qualitativamente determinada conforme a variação do volume do meio, como mostrado da Tabela 1 (DUSSÁN et al., 2016).

Tabela 1 - Variáveis do planejamento fatorial

\begin{tabular}{c|c|c|c}
\hline Variáveis & $\mathbf{- 1}$ & $\mathbf{0}$ & $\mathbf{1}$ \\
\hline $\mathrm{X} 1$ - Volume do meio $(\mathrm{mL})$ & 25 & 50 & 75 \\
$\mathrm{X} 2$ - Concentração celular $\left(\mathrm{gL}^{-1}\right)$ & 1 & 5 & 10 \\
$\mathrm{X} 3$ - Farelo de arroz $\left(\mathrm{gL}^{-1}\right)$ & 0 & 10 & 20 \\
\hline
\end{tabular}




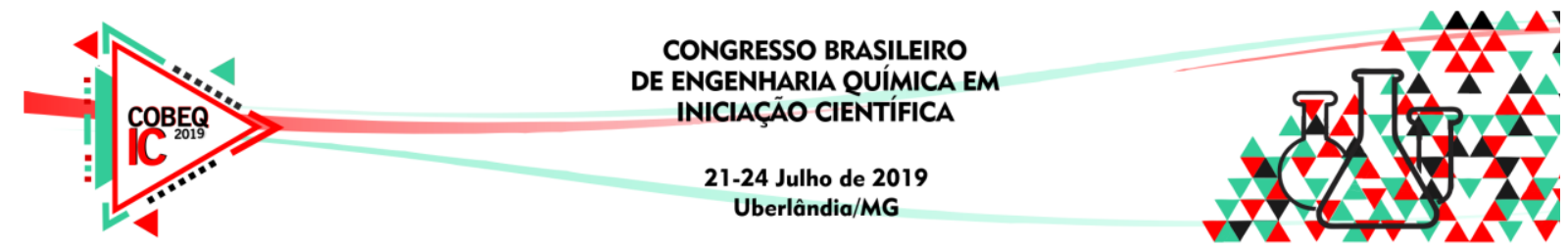

Os níveis das variáveis foram definidos e relacionados com as faixas -1 correspondente ao nível inferior, 0 correspondente ao nível intermediário e +1 ao nível superior (DUSSÁN et al., 2016).

\subsection{Fermentação}

Os cultivos foram realizados em frascos de Erlenmeyers de $125 \mathrm{~mL}$ contendo $50 \mathrm{~mL}$ de meio composto por glicose $\left(50\right.$ g. $\left.\mathrm{L}^{-1}\right)$, extrato de levedura $\left(3 \mathrm{~g} . \mathrm{L}^{-1}\right)$, e sulfato de amônio foram $\left(1 \mathrm{~g} . \mathrm{L}^{-1}\right)$, em todos os meios de fermentação. Nesta etapa foi realizado o planejamento fatorial $2^{3}$ de acordo com o item 2.2, variando a concentração de farelo de arroz, concentração celular e volume de meio. Este último representa a quantidade de oxigênio disponível, sendo que um maior volume de meio $(75,0 \mathrm{~mL})$ representa uma menor disponibilidade de oxigênio e um menor volume de meio $(25,0 \mathrm{~mL})$ representa uma maior disponibilidade de oxigênio, influenciando assim a rota metabólica da levedura. Os Erlenmeyers foram incubados no shaker (New Brunswick Scientific CO. INC. series 25) por 60 horas à temperatura de $25^{\circ} \mathrm{C}$ sob agitação de $200 \mathrm{rpm}$, e uma alíquota foi coletada a cada 12 horas para análises posteriores (CARNEIRO, 2011).

\subsection{Determinação de açúcares, etanol e glicerol}

As concentrações de glicose, etanol e glicerol foram determinadas por cromatografia líquida de alta eficiência (CLAE), utilizando-se o cromatógrafo Shimadzu (Shimadzu, UFLC). Foram calculados a taxa de produtividade volumétrica (Qp) e rendimento de etanol $\left(Y_{p / s}\right)$ e a eficiência $(\boldsymbol{\eta})$.

\section{RESULTADOS E DISCUSSÃO}

Com os resultados obtidos, foi possível observar que os ensaios 1, 2, 5 e 6 obtiveram maior produção de etanol em 24 horas de fermentação, sendo esses realizados com uma concentração celular de 1 g.L. $\mathrm{L}^{-1}$. Por outro lado, os ensaios 3, 4, 7 e 8 foram realizados com uma concentração celular de 10 g.L $\mathrm{L}^{-1}$ e obtiveram maior produção de etanol em 12 horas de fermentação (Tabela 2). Isso pode ser explicado em relação ao crescimento celular, uma vez que foi possível observar que nos ensaios 1,2, 5 e 6 houve um crescimento celular até 24 horas, enquanto nos ensaios 3, 4, 7 e 8 a concentração celular permaneceu praticamente constante durante todo o tempo de fermentação (Gráfico 1 (a) e 1 (b)).

Pode-se inferir também de acordo com a Tabela 2 que os ensaios 2 e 4 foram os que obtiveram maior produção de etanol $\left(26,36 \mathrm{~g} . \mathrm{L}^{-1}\right.$ e $26,91 \mathrm{~g} . \mathrm{L}^{-1}$, respectivamente), sendo que esses ensaios foram realizados na ausência de farelo de arroz.

Observou-se também que houve uma variação significativa na conversão de substrato Yps $\left(0,24 \mathrm{~g} \cdot \mathrm{g}^{-1}\right.$ a $\left.0,74 \mathrm{~g} \cdot \mathrm{g}^{-1}\right)$ e na produtividade volumétrica Qp $\left(0,15 \mathrm{~g} \cdot \mathrm{L}^{-1} \cdot \mathrm{h}^{-1}\right.$ a $\left.1,29 \mathrm{~g} \cdot \mathrm{L}^{-1} \cdot \mathrm{h}^{-1}\right)$, conforme apresentado na Tabela 2. 


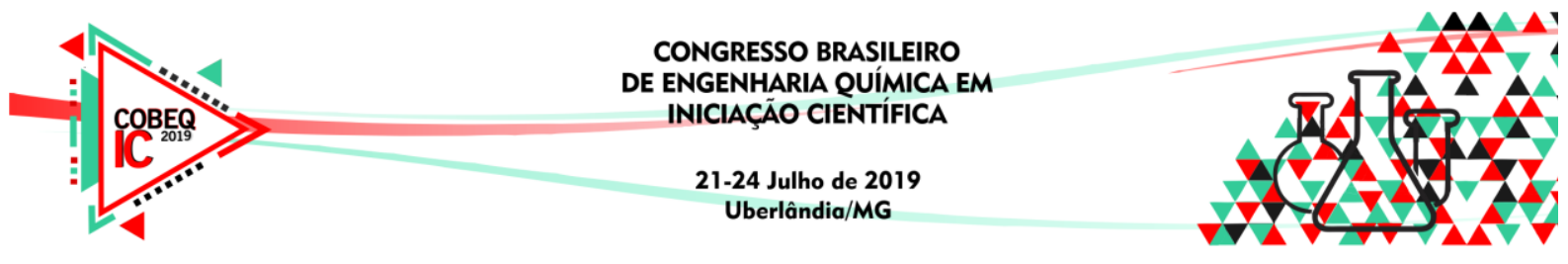

Tabela 2 - Valores utilizados e codificados no planejamento fatorial completo $2^{3}$, com 3 repetições no ponto central para a avaliação do efeito do volume do meio (X1), concentração celular (X2) e a suplementação de farelo de arroz (X3) para a produção de etanol

\begin{tabular}{|c|c|c|c|c|c|c|c|c|c|c|}
\hline Ensaio & $\begin{array}{c}\mathrm{V} . \\
\text { (X1) }\end{array}$ & $\begin{array}{c}\mathrm{C} . \\
\text { (X2) }\end{array}$ & $\begin{array}{l}\text { F.A. } \\
\text { (X3) }\end{array}$ & $\begin{array}{c}\text { Tempo } \\
\text { (h) }\end{array}$ & $\begin{array}{c}\text { Consumo } \\
\text { de } \\
\text { Glicose } \\
(\%)\end{array}$ & $\begin{array}{l}\text { Etanol } \\
\left(\mathrm{g} . \mathrm{L}^{-1}\right)\end{array}$ & $\begin{array}{c}\text { Glicerol } \\
\left(\mathrm{g} . \mathrm{L}^{-1}\right)\end{array}$ & $\begin{array}{c}Y_{p / s} \\
\left(\mathrm{~g} \cdot \mathrm{g}^{-1}\right)\end{array}$ & $\begin{array}{c}\text { Qp } \\
\left(\mathrm{g} \cdot \mathrm{L}^{-}\right. \\
\left.{ }^{1} \cdot \mathrm{h}^{-1}\right)\end{array}$ & $\eta(\%)$ \\
\hline \multirow[t]{2}{*}{1} & 25,0 & 1,0 & 0,0 & 12 & 62,93 & 12,70 & 0,01 & 0,31 & 0,50 & 91,80 \\
\hline & & & & 24 & 87,60 & 19,58 & 1,54 & 0,34 & 0,45 & 96,70 \\
\hline \multirow[t]{2}{*}{2} & 75,0 & 1,0 & 0,0 & 12 & 79,49 & 19,20 & 0,01 & 0,47 & 0,73 & 84,47 \\
\hline & & & & 24 & 100,0 & 26,36 & 1,57 & 0,49 & 0,58 & 50,07 \\
\hline \multirow[t]{2}{*}{3} & 25,0 & 10,0 & 0,0 & 12 & 100,0 & 23,10 & 0,01 & 0,43 & 0,98 & 84,31 \\
\hline & & & & 24 & 100,0 & 15,89 & 3,02 & 0,26 & 0,15 & 50,98 \\
\hline \multirow[t]{2}{*}{4} & 75,0 & 10,0 & 0,0 & 12 & 100,0 & 26,91 & 3,53 & 0,74 & 1,29 & 98,92 \\
\hline & & & & 24 & 100,0 & 23,01 & 3,08 & 0,61 & 0,45 & 96,82 \\
\hline \multirow[t]{2}{*}{5} & 25,0 & 1,0 & 20,0 & 12 & 100,0 & 19,89 & 1,37 & 0,24 & 0,40 & 47,06 \\
\hline & & & & 24 & 100,0 & 19,94 & 1,29 & 0,28 & 0,43 & 54,90 \\
\hline \multirow[t]{2}{*}{6} & 75,0 & 1,0 & 20,0 & 12 & 99,27 & 24,65 & 2,85 & 0,34 & 1,12 & 66,91 \\
\hline & & & & 24 & 100,0 & 24,69 & 2,93 & 0,33 & 0,58 & 64,70 \\
\hline \multirow[t]{2}{*}{7} & 25,0 & 10,0 & 20,0 & 12 & 100,0 & 20,25 & 2,85 & 0,43 & 0,97 & 84,37 \\
\hline & & & & 24 & 100,0 & 18,50 & 2,89 & 0,39 & 0,45 & 77,20 \\
\hline \multirow[t]{2}{*}{8} & 75,0 & 10,0 & 20,0 & 12 & 100,0 & 23,56 & 3,67 & 0,50 & 1,13 & 97,13 \\
\hline & & & & 24 & 100,0 & 23,18 & 3,66 & 0,49 & 0,56 & 95,50 \\
\hline \multirow[t]{2}{*}{9} & 50,0 & 5,0 & 10,0 & 12 & 100,0 & 25,00 & 3,22 & 0,42 & 1,13 & 81,64 \\
\hline & & & & 24 & 100,0 & 22,78 & 3,03 & 0,38 & 0,54 & 74,41 \\
\hline \multirow[t]{2}{*}{10} & 50,0 & 5,0 & 10,0 & 12 & 100,0 & 23,86 & 2,32 & 0,57 & 1,16 & 98,72 \\
\hline & & & & 24 & 100,0 & 22,91 & 3,32 & 0,53 & 0,53 & 96,12 \\
\hline \multirow[t]{2}{*}{11} & 50,0 & 5,0 & 10,0 & 12 & 100,0 & 23,32 & 3,37 & 0,44 & 1,04 & 85,99 \\
\hline & & & & 24 & 100,0 & 23,32 & 3,26 & 0,44 & 0,52 & 85,99 \\
\hline
\end{tabular}

Gráficos 1 (a) e 1 (b) - Crescimento celular para as concentrações de 1 g.L. $L^{-1}$ e 10 g.L ${ }^{-1}$

(a)

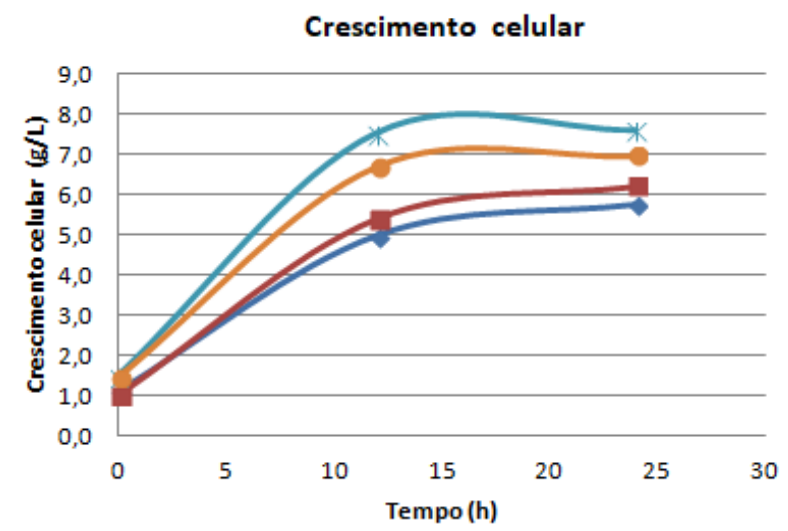

(b)

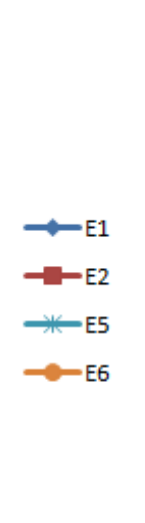

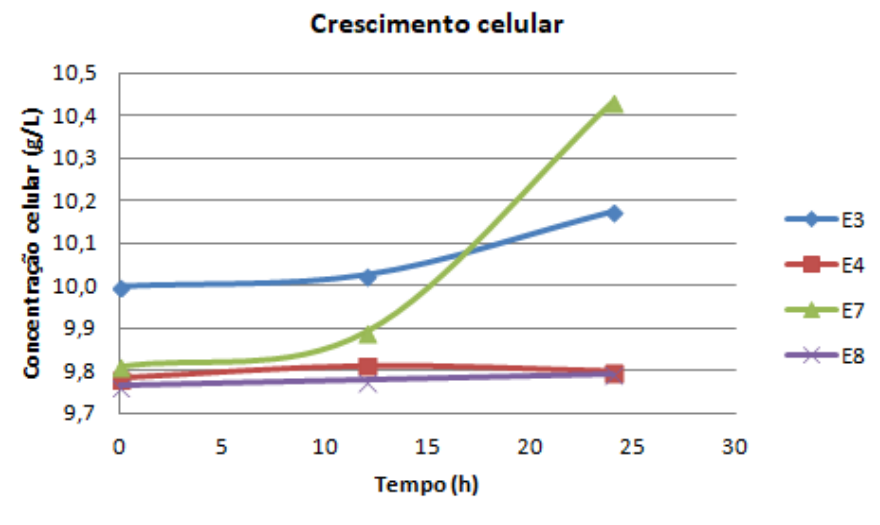

Por fim, foi possível constatar que houve produção de glicerol em um tempo de 24 horas de fermentação e que após esse tempo a concentração do mesmo diminuiu. A produção 


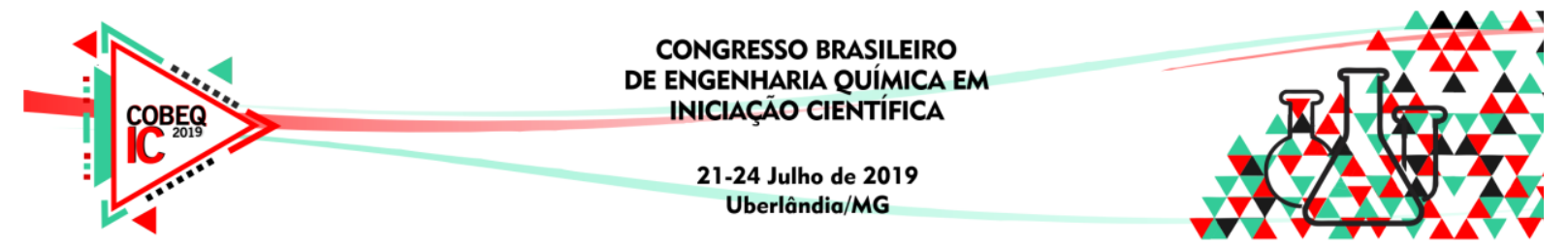

de glicerol pode ter ocorrido devido à exposição da levedura a uma condição de estresse físico (pressão osmótica), em resposta a isso a mesma sintetiza o glicerol para proteger suas estruturas moleculares (MELO, 2006).

Foi realizada a análise estatística com auxílio do programa Statsoft Statistica ${ }^{\circledR}$ v12.5, utilizando a ferramenta de análise de experimentos para os tempos de 12 e 24 horas. A partir dessa análise foi possível determinar a significância dos fatores utilizados (concentração de células, volume de meio e farelo de arroz), assim como a interação entre eles, sobre o fator de conversão de substrato em etanol $\left(\mathrm{Y}_{\mathrm{p} / \mathrm{s}}\right)$. Essa análise foi realizada com um nível de confiança de $95 \%$, assim, obtiveram-se os gráficos de pareto para os tempos de 12 e 24 horas, nos quais foi possível avaliar a significância dos fatores citados anteriormente (Gráfico 2 (a) e 2 (b), respectivamente).

Gráfico 2 - Gráfico de pareto dos efeitos principais e de interações dos fatores avaliados na composição do meio de fermentação para a produção de etanol por Saccharomyces cerevisiae nos tempos de 12 horas (a) e 24 horas (b), sobre o fator de conversão de substrato em etanol (Yps)

(a)

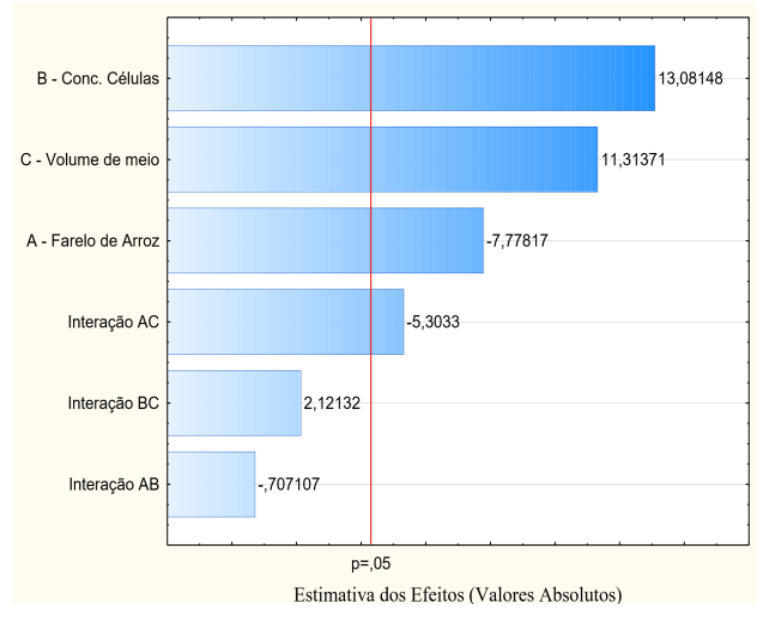

(b)

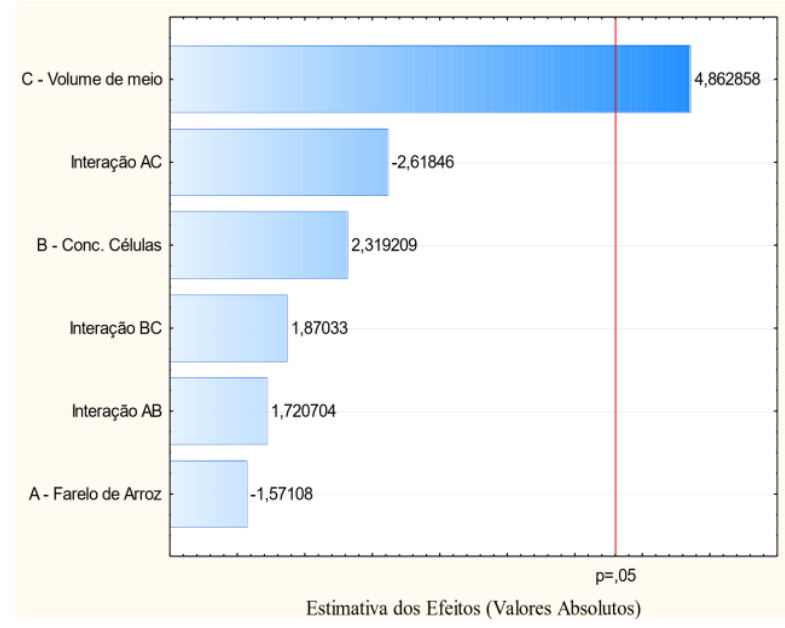

A partir dos gráficos de pareto, foi possível observar que o volume de meio foi o fator de maior significância, os ensaios que obtiveram maior produção de etanol foram realizados com maior volume de meio, ou seja, 75,0 mL. Isso pode ser explicado pelo metabolismo das leveduras, pois segundo Melo (2006), as leveduras possuem duas vias metabólicas: a respiração celular e a fermentação. Sendo que em um meio com menos disponibilidade de oxigênio a fermentação é dominante.

O valor negativo do fator farelo de arroz no Gráfico 2 (a) possibilitou constatar estatisticamente que a presença do mesmo não contribuiu para a conversão de substrato em etanol $\left(\mathrm{Y}_{\mathrm{p} / \mathrm{s}}\right)$, porém isso não inviabiliza sua utilização como suplemento, apenas mostra que as concentrações de 10 g.L $\mathrm{L}^{-1}$ e $20 \mathrm{~g} . \mathrm{L}^{-1}$ de farelo de arroz não se mostraram efetivas na produção de etanol, utilizando a levedura Saccharomyces.cerevisiae.

\section{CONCLUSÃO}

A concentração de célula de $10 \mathrm{~g} . \mathrm{L}^{-1}$ obteve a maior produção de etanol nas primeiras 12 horas, porém os valores de produção de etanol utilizando $1 \mathrm{~g} . \mathrm{L}^{-1}$ de concentração celular 


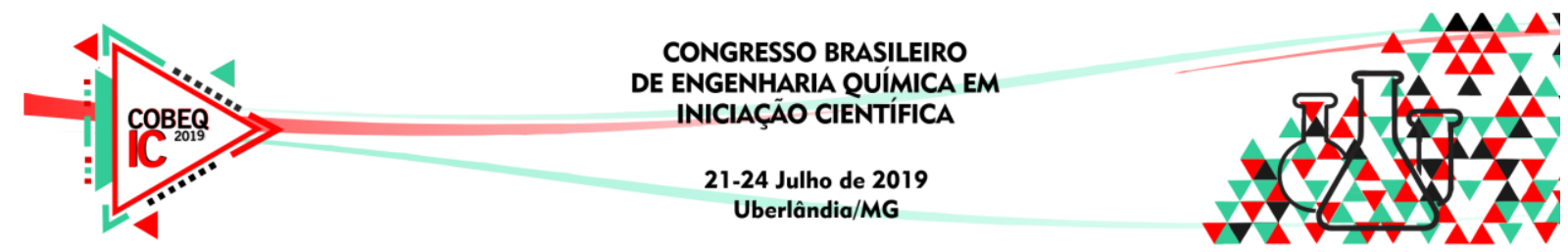

não apresentaram diferenças significativas em um tempo de 24 horas. Dessa forma, ambas as concentrações podem ser utilizadas para a obtenção de etanol. No entanto, para minimizar a utilização de uma concentração de células maior $\left(10\right.$ g. $\left.\mathrm{L}^{-1}\right)$, pode-se utilizar uma quantidade de células menor $\left(1\right.$ g. $\left.\mathrm{L}^{-1}\right)$ estendendo o tempo de fermentação para 24 horas, pois a obtenção de meios de fermentação com maior concentração de células demanda maior gasto de material. Em um meio com menor quantidade de oxigênio, ou seja, em um meio de 75,0 mL, foi atingida uma maior conversão de substrato em etanol, mostrando que em uma condição com menor aeração a produção de etanol é favorecida. $\mathrm{O}$ farelo de arroz sendo utilizado como suplemento não favoreceu a produção de etanol utilizando a $S$. cerevisiae nas concentrações celulares de $10 \mathrm{~g} . \mathrm{L}^{-1}$ e $20 \mathrm{~g} . \mathrm{L}^{-1}$.

\section{REFERÊNCIAS}

CARNEIRO, Lívia Melo. Avaliação de estratégias de cultivo da levedura Pichia stipitis em hidrolisado hemicelulósico de palha de arroz visando à produção de etanol. $2011.175 \mathrm{f}$. Tese (Doutorado). Programa de Pós- Graduação em Biotecnologia Industrial na área de conversão de biomassa. Universidade de São Paulo, Lorena, 2011.

DUSSÁN, Kelly J., et al. Evaluation of oxygen availability on ethanol production from sugarcane bagasse hydrolysate in a batch bioreactor using two strains of xylosefermenting yeast. Departamento de Biotecnologia, Escola de Engenharia de Lorena, São Paulo, 2016.

FERRARI, Fernanda C. dos Santos. Fatores operacionais e cinética do processo fermentativo para otimização da produção de etanol em escala industrial. 2013.74 f. Dissertação (Mestrado). Mestrado em Microbiologia Agropecuária. Universidade Estadual de São Paulo. Jaboticabal, 2013.

GONÇALVES, K. Y. et al. Processo produtivo do etanol hidratado a partir da cana de açúcar. 2015. Disponível em: <http://www.fecilcam.br/anais/ix_eepa/data/uploads/11agroindustria/11-01.pdf > . Acesso em: Setembro, 2018.

MELO, H. F. Resposta ao estresse acido em leveduras da fermentação alcoólica industrial. Tese de Mestrado, Recife/PE, p.118, 2006.

MOURA, M. Produção de etanol e xilitol a partir de D-xilose por linhagens Candida (Spathaspora) materiae. Dissertação (Mestrado em Microbiologia) - Universidade Federal de Minas Gerais- UFMG, 2015.

WALTER, Melissa ; MARCHEZAN, Enio; AVILA, Luis Antonio de. Arroz: composição e características nutricionais. Ciência Rural. v.38, n.4, p.1184-1192. Santa Maria. Julho, 2008. 